\title{
Communicate to Illuminate: State-of-the-art and Research Challenges for Visible Light Communications
}

\author{
Ozgur Ergul, Ergin Dinc, Ozgur B. Akan \\ Next-generation and Wireless Communications Laboratory \\ Department of Electrical and Electronics Engineering \\ Koc University, Istanbul, 34450 Turkey
}

\begin{abstract}
In the near future, the available radio-frequency (RF) bandwidth will not be sufficient to meet the ever increasing demand for wireless access. Visible light communication (VLC) is an alternative method to reduce the burden of RF-based communication, especially in indoor communications. 70\% of the communication is indoors, and light emitting diode (LED) arrays are spreading for illumination purposes thanks to their low energy and higher lifetime. VLC can be realized as a secondary application in LED arrays that are placed for lighting. In this way, some of the wireless traffic can be sent using light, with less cost and less carbon footprint. For these reasons, VLC attracts significant research interests. We provide an extensive survey of the current literature by outlining challenges and future research areas in order to facilitate future research in this area.
\end{abstract}

Keywords: Visible light communications, Optical communications, OFDM, MIMO, Modulation

\section{Introduction}

The demand for wireless access has become so prevalent that it is possible to consider wireless connectivity as one of the basic commodities like electricity. This rapidly growing demand resulted in ubiquitous deployment of wireless systems. Eventually, the limited wireless spectrum got heavily congested and solutions increasing spectrum efficiency, such as spectrum reuse got to a point that even small cells (pico/femtocells) will not be able to help with covering the huge demand. Recent studies predict that by 2017 , more than 11 exabytes of data traffic will have to be transferred through mobile networks every month [1]. To be able to meet this demand, the research community began looking for solutions that target alternative portions of the spectrum. VLC is one of the promising alternative that aims to provide a communication medium by using the existing illuminating devices.

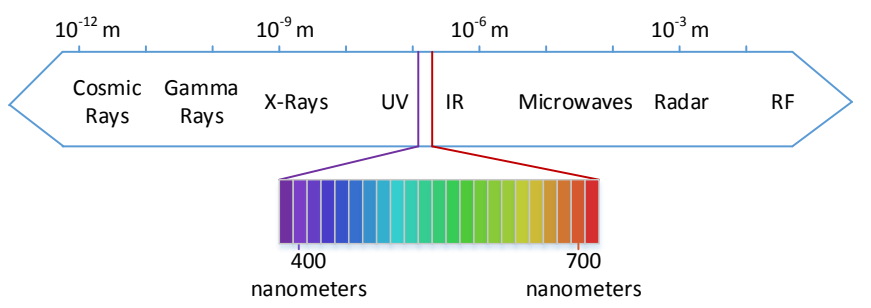

Figure 1: Electromagnetic spectrum and visible light region.

With the improvements in LED technologies, it is possible

Email addresses: ozergul@ku. edu.tr (Ozgur Ergul), edinc@ku.edu.tr (Ergin Dinc), akan@ku.edu.tr (Ozgur B. Akan) to modulate light in high frequencies such that human eye cannot detect. Due to their lower cost, higher lifetime and lower power consumption, LEDs are expected to replace conventional incandescent and fluorescent lamps in the near future. This enables the use of LEDs for both illumination and communication, making VLC an economic and ubiquitous data transmission solution.

In Figure 1, we depict the electromagnetic spectrum. Visible light region corresponds roughly to the portions where the wavelength is between 400 to 700 nanometers. Unlike radio waves, electromagnetic waves in the visible light wavelength are not harmful for the human body. Moreover, the visible light portion of the spectrum is not regulated. This opens up a huge bandwidth for communication, which can be utilized in a wide range of applications.

In this paper, we aim to capture the state-of-the-art for this timely and exciting field by discussing the open research issues. The remainder of the paper is organized as follows. In Section 2 , we explain the advantages of VLC and provide comparisons with other wireless communication technologies that use lower parts of the spectrum. In Section 3, we summarize the historical evolution of VLC technology and list the standardization efforts. We review previous work and open research issues on the transmitter LED technologies and modulation schemes in Sections 4. Section 5 includes the channel modeling technique for optical paths. The optical receivers and MIMO systems for VLC are summarized in Section 6. We examine the research on medium access control (MAC) and network layers as well as multiple access schemes in Section 7. We list the potential application areas for VLC in Section 8. We present our concluding remarks in Section 9. 
Table 1: Comparison of wireless communication technologies

\begin{tabular}{|c|c|c|c|c|}
\hline Type & Technology & Range & Rate & Mobility \\
\hline \multirow{6}{*}{ RF } & Wi-Fi 2.4 GHz & Indoor $70 \mathrm{~m}$, outdoor $250 \mathrm{~m}$ & $65 \mathrm{Mbps}$ & Low \\
\hline & Wi-Fi 5 GHz & Indoor $35 \mathrm{~m}$ & $780 \mathrm{Mbps}$ & Low \\
\hline & 3G HSPA & Depending on the cell type (pico-macrocell) up to $100 \mathrm{~km}$ & 42 Mbps & High \\
\hline & \multirow{2}{*}{ 4G } & \multirow{2}{*}{ Depending on the cell type (pico-macrocell) up to $100 \mathrm{~km}$} & Up to 1 Gbps & High \\
\hline & & & Up to $100 \mathrm{Mbps}$ & Low \\
\hline & MM-wave (60 GHz) & A few hundreds of meters & 7 Gbps & Low \\
\hline \multirow{2}{*}{ Optical } & IR & $1 \mathrm{~m}$ & 1Gbps & None \\
\hline & VLC & Up to $10 \mathrm{~m}$ & Up to 3 Gbps & Low \\
\hline
\end{tabular}

\section{Why VLC?}

In this section, we detail the features provided by VLC and explain why it is an important alternative to RF communication technologies. Below, we list the prominent advantages offered by VLC.

\section{- Cost efficiency}

- Energy efficiency

- Unregulated large bandwidth

Today, RF technology is mature. Yet, while a Bluetooth module that provides $1 \mathrm{Mb} / \mathrm{s}$ costs around \$5 [2], VLC links can transmit at $50 \mathrm{Mb} / \mathrm{s}$ with an approximate cost of $\$ 1.7$ [3]. Furthermore, LEDs used in VLC are also utilized for illumination. Therefore, the exact cost is even less.

LEDs used in VLC are highly efficient devices that use at least $75 \%$ less energy and last 25 times longer than incandescent lighting [4]. Since energy used by LEDs is mainly needed for illumination, VLC is extremely energy efficient. Estimations for the United States indicate greatest potential improvement on energy savings will be achieved upon the widespread use of LED lighting. According to these estimations, widespread use of LEDs by 2027 can save about 348 TerraWatt-hour of electricity compared to no LED use. This is an enormous amount of energy saving that is equal to the annual electrical output of 44 large electric power plants. Overall, this corresponds to total savings of more than $\$ 30$ billion [4].

VLC uses the spectrum between $385 \mathrm{THz}$ and $800 \mathrm{THz}$. Considering the huge bandwidth, the potential data carrying capacity of VLC is thousands of times larger than the RF portion of the spectrum [5].

On the other hand, it is difficult to install new cables to the lighting equipment on the ceiling. Fortunately, power line communications (PLC) enable use of electric cables for communication. PLC also enables the use of power outlets to be used as ports. This alleviates the need to install new communication cables to make VLC work. PLC specifications have been consolidated into two standards, i.e. IEEE 1901 [6] and ITU-T G.9960/61 [7] in 2009 and 2010, respectively. Since standardization for both VLC and PLC are complete, there is a strong incentive to investigate the integration of these technologies. In [8], authors point out the potential for this unification and lay out a few promising areas such as MIMO and relaying.
VLC has other advantages but these are more apparent when combined to alternative communication technologies. In the following, we lay out a comparison of VLC with Infrared (IR) and RF communications and point out the advantages provided by VLC. We provide a comparison of VLC with other wireless communication technologies in Table 1.

\subsection{Comparison of VLC with IR Communication}

VLC has two major advantages over IR. One is related to safety issues and the other is about ease of deployment. Most of the Infrared emitting diodes use the $800 \mathrm{~nm}$ - $960 \mathrm{~nm}$ wavelength range. A number of problem may arise if radiation within these wavelengths comes into direct contact with the eye, such as athermal retina hazard and thermal injury risk of the cornea as well as possible delayed effects on the lens of the eye (cataractogenesis). Therefore, transmission power for infrared devices are limited by safety standards such as International Electrotechnical Commissions (IEC) IEC 60825-1 Safety of laser products, and IEC 62471 Photobiological safety of lamps and lamp systems.

VLC uses visible light LEDs which are expected to replace the conventional incandescent and fluorescent lamp since they have lower power consumption, high efficiency and longer lifetime $[9,10]$. Therefore, the transmitters for VLC will mostly be readily available. Furthermore, technologies such as PLC enable use of existing lighting infrastructure as back-haul in existing installations. For new installations, new technologies such as Power over Ethernet (PoE) may be used.

Room illumination must meet certain minimum levels according to the standards. For example, the international standard on Lighting of indoor work places, ISO/CIE 8995.1 recommends a minimum illuminance of 200 lux in areas where continuous work is carried out [11]. To meet these illumination levels, distributed ceiling installations are envisioned. Such deployment of LEDs ensure a dominant line-of-sight (LOS) component, resulting in very high signal-to-noise ratio (SNR) $(>60$ $\mathrm{dB}$ through the entire room) [12]. This permits simpler receiver structures for VLC compared to IR. For example, due to this large SNR, the receiver does not need to narrow the field-ofview (FOV) [13].

\subsection{Comparison of VLC with RF Communication}

Even though both RF and VL communications use electromagnetic waves, they have very different inherent properties. 
Visible light does not interfere with electronic devices as RF waves do. Therefore, VLC may be more suitable for applications where sensitive electronic devices are used, such as hospitals, chemical plants, and airplanes.

Recent studies indicate that more than $70 \%$ of wireless traffic originates indoors [14]. Even though RF waves penetrate walls, signal propagation is degraded. On the one hand, this attenuated propagation limits data rates of intended users. On the other hand, since transmission is not strictly confined to the intended area, security of the links may be compromised by eavesdropping malicious users. VLC provides the desired answers to both problems. Since most indoor environments are illuminated, VLC can provide the required coverage. Since visible light cannot penetrate walls, links can be kept confidential.

\subsection{Comparison of VLC with Small Cells and HetNets}

A comparison of frequency reuse in VL and RF communications is important. RF spectrum is already subject to an aggressive spatial reuse to increase the spectrum efficiency as much as possible. New approaches such as small cells and HetNets are widely seen as the future of mobile communications. A HetNet is a heterogeneous network that support $3 \mathrm{G}$ or $4 \mathrm{G}$ for back-haul and shorter range communication technologies such as $\mathrm{Wi}-\mathrm{Fi}$ for offloading localized network traffic. Due to cell-size reduction, by year 2007, the system spectral efficiency was increased by a factor of 2700 compared to 50 years earlier [15]. Due to high directivity and easier confinement of visible light, even smaller VLC attocells that provide hundreds of times larger capacity increase is possible [16].

An efficient HetNet should use the most suitable link for each specific task, e.g., fiber optic links are for high speed backhaul connectivity, RF links support mobility and provide wide coverage and VLC can be used when high data rates and secure communication is needed. With such a vision in mind, VLC is evolving towards a new paradigm called Li-Fi (Light Fidelity) to complement RF based Wi-Fi (Wireless Fidelity) networks [17].

\section{Evolution of VLC Technology and Standardization}

Research on VLC using LEDs originated at Nakagawa Laboratory, in Keio University, Japan in 2003. The same year, Visible Light Communications Consortium (VLCC) [18] was funded. Since then, there has been an increasing widespread interest. In 2006, combining PLC with VLC to provide broadband access for indoor applications was proposed by researchers from CICTR at Penn State [19]. The idea was to handle backhaul data transmission through PLC and use VLC to cover the local area network (LAN).

Standardization began in 2007, when the Japan Electronics and Information Technology Industries Association (JEITA) issued two visible light standards, JEITA CP-1221 and JEITA CP-1222, based on VLCC proposals. VLCC began cooperation with the Infrared Data Association (IrDA) and the Infrared Communication Systems Association (ICSA) in 2008. The following year, a VLCC specification that extends the IrDA physical layer was announced.
IEEE P802.15 Working Group for Wireless Personal Area Networks (WPANs) finished standardization of physical (PHY) and MAC layers for short-range optical wireless communications using visible light in 2011 [20]. The standard describes the methods to merge lighting and data communication for wireless personal area networks (WPAN), to deliver data rates sufficient for audio and video multimedia services. It offers three PHY types that provide data rates from $11.67 \mathrm{Kbps}$ to $96 \mathrm{Mbps}$. The first two types support on-off keying (OOK) and variable pulseposition modulation (VPPM). The third type, PHY III, provides data rates at the range 12 to $96 \mathrm{Mbps}$ and uses multiple light sources operating at different frequencies. A modulation format called color shift keying (CSK) is used. The supported modulation types provide different trade-offs with respect to data rates and required dimming conditions [21]. By using OOK under dimming, constant range and variable data rate is provided by inserting compensation time. On the other hand, VPPM provides constant data rate and variable range by varying the pulse width [22]. Run length limited (RLL) line codes are used to remove flicker and clock and data recovery (CDR) detection problems by controlling the maximum amount of consecutive $1 \mathrm{~s}$ and $0 \mathrm{~s}$. Also various forward error correction (FEC) schemes are supported.

The established standards provide stability in the field and enable faster commercialization of VLC. However, the research on VLC is rapidly evolving. By the end of 2010, data rates up to $500 \mathrm{Mbps}$ for a transmission distance of 5 meters were demonstrated by a combined research team of Siemens and Fraunhofer Institute for Telecommunications [23]. A commercial bidirectional RGB LED VLC system called MOMO was launched in October 2014. The system is capable of data rates up to 300 Mbps with a range of 25 feet and is PLC compatible [24].

With the ongoing research, the established standards such as IEEE 802.15.7 will evolve. For example, a recently formed study group is working on an amendment to IEEE 802.15.7. The aim is to enable scalable data rate, positioning/localization, message broadcasting, etc. for optical camera communications [25], [26]

\section{VLC Transmiters}

Both RF and VLC communication utilize electromagnetic radiation to transmit data. However, inherent properties of them significantly differ [16]. The waves in the visible range cannot penetrate into objects. Therefore, most of the VLC applications require LOS for high data rate applications. Data communication in VLC is provided via light, thus transmitted signal in VLC is required to be positive and real. [27]. For these reasons, well-developed theories for RF communications cannot be directly used for VLC. In this section, we review LED technologies and modulation techniques for VLC.

\subsection{LED Technology}

The key enabling technology for VLC is the increasing usage of white LEDs in commercial lighting applications. It is predicted that the dominant lighting method will be LEDs in the 
Table 2: Properties of LEDs.

\begin{tabular}{|c|c|l|l|}
\hline Type & Data rate Mbps $(\approx)[35]$ & \multicolumn{1}{|c|}{ Advantageous } & \multicolumn{1}{c|}{ Disadvantageous } \\
\hline White LED & 40 & Low cost, availability & Low data rates \\
\hline RGB LED & 100 & Higher data rates, suitable for CSK & Higher cost, more complex design \\
\hline RCLED & 500 & Very high data rates & Challenging to manufacture, high cost \\
\hline
\end{tabular}

near future. LEDs have lower power consumption, and longer lifetime compared to the conventional lighting systems such as incandescent or fluorescent lamps. White LEDs can be also used for communication purposes as transmitters. Since white LEDs are commercially available with low costs, most of the recent studies on VLC consider the white LEDs as transmitter $[10,28,29,30,31,32]$. However, other types of LEDs are also promising as summarized in Table 2 because higher bandwidths and data rates can be provided with more complex LEDs such as Red-Green-Blue (RGB) LEDs, and $\mu$ LEDs [37, 38].

In modern white LEDs, single LED with yellow phosphor is utilized to emit white light. The emission power and bandwidth of LEDs can significantly change with technology and manufacturing methods. Since the yellow phosphor, which is utilized for emitting white light, has a slow response, the bandwidth of white LEDs is limited to $\approx 3 \mathrm{MHz}$ [28, 34]. However, the capacitance and area of the blue region is higher, thus blue light is more promising for communication applications. For this reason, an optical blue filter can be placed at the receiver [34] at the expense of optical power to increase the bandwidth to $10 \mathrm{MHz}$ range $[32,34]$. In addition, white LEDs can suffer from color shifts [35]. Therefore, design of high data rate applications with white LEDs is very challenging.

In addition to white LEDs, RGB LEDs can be utilized to transmit information in VLC [33]. RGB LEDs have relatively higher response times, thus they can reach up to a few tens of $\mathrm{MHz}$. They are expected to achieve data rates up to $100 \mathrm{Mbps}$ compared to white LEDs that can reach up to $40 \mathrm{Mbps}$. RGB LEDs enable color shift keying (CSK) as suggested in [36]. Since CSK can reach constellation sizes up to 64 symbols, it is more promising for high data rate applications. Furthermore, all three colors can be separately coded to create parallel channels. For example, two of the colors can be used to transmit real and imaginary part of orthogonal-frequency-division-multiplexing (OFDM) signals and the other one can be utilized to control whiteness. RGB LEDs are also susceptible to color change during modulations [35]. Transmitting information with RGB LEDs is more complex and expensive compared to white LEDs however, RGB LEDs offer considerable data rate improvement over white LEDs.

$\mu$ LEDs also attract significant research attention. Resonantcavity LEDs (RCLED) based on AlInGaN LEDs provide the lowest-loss transmission window around $520 \mathrm{~nm}$ and bandwidth levels up to $330 \mathrm{MHz}$ [39]. [40] reports that free-space modulated light can be reach up to $200 \mathrm{Mbps}$ data rate [40]. In addition, RCLEDs can provide highly directional output and high data rates up to $500 \mathrm{Mbps}$ [35], but the fabrication of RCLEDs is challenging [41]. In addition, [37] provides a transmitter design with AlInGaN-based micro-pixellated LEDs. In- stead of a single LED, an array of $\mu$ LEDs are utilized to provide high bandwidths up to $245 \mathrm{MHz}$ per pixel. In [38], a blue (450 $\mathrm{nm}) \mathrm{GaN}$-based micron-size LED array is utilized to provide $1.5 \mathrm{Gbps}$ data rate. Therefore, $\mu$ LEDs provide higher bandwidths and data rates at the expense of complexity and cost. The advancement in LED manufacturing especially in $\mu$ LEDs has high potential to increase the performance of VLC systems.

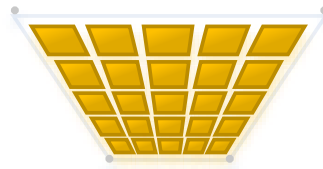

(a)

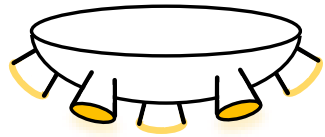

(b)
Figure 2: (a) Rectangular transmitter and, (b) angle-diversity transmitter.

Up to now, we mostly considered single LEDs in transmit arrays. Design of transmit arrays is also significant for the performance of VLC. For illumination purposes, LED arrays are utilized because single LED can not provide enough illumination. Generally, rectangular LED arrays are utilized for illumination as in Figure 2(a). [10, 42] also utilize rectangular arrays for communication purposes. However, design of the transmit arrays can be changed for some applications. For example, circular LED arrays can be utilized in desk lamps with fewer number of LEDs. Angular diversity systems are utilized in VLC to cluster users with low inter-cell interference [29, 43, 44, 45]. Figure 2(b) shows an angle diversity receiver. Figure 2(b). In this way, OFDMA VLC employments can provide higher reuse factors.

To sum up, there are two main challenges in utilization of LEDs as transmitter:

- Limited bandwidth: Bandwidth can be extended with complex type of LEDs.

- Nonlinearity: Nonlinear relationship between current and optical power in LEDs becomes challenging in high data rate applications. It is inevitable.

\subsection{Modulation Schemes}

In VLC, information is carried with light intensity, thus modulated signals have to be both unipolar and real [27]. For this reason, well-studied advanced RF communication techniques are not directly applicable to VLC. More importantly, dual-use of LEDs for both illumination and communication purposes introduces significant challenges: dimming and flicker control. Illumination level of a room can be changed manually. Therefore, modulation schemes for VLC should support dimming 
Table 3: Comparison of Modulation Schemes.

\begin{tabular}{|c|c|c|c|l|}
\hline Modulation & Dimming Control & Flicker Effect & Data Rate & Properties \\
\hline OFDM & No & Medium & High & Eliminate ISI, PWM for dimming control \\
\hline VPPM & Yes & Medium & Low & Spectral efficiency problem, easy to implement \\
\hline MEPPM & Yes & Low & Moderate & More susceptible for ISI \\
\hline CSK & Yes & Low & High & Color shift problem \\
\hline MM & Yes & Low & Moderate & Constant color control \\
\hline SM & No & High & Moderate & Provide diversity, severe problems \\
\hline
\end{tabular}

control to achieve desired data rate levels even at low illumination levels. Fluctuations of the light intensity can be harmful for human eye during long exposure periods [46, 47]. Therefore, modulation techniques are required to employ flicker control not to affect human health. For this purpose, IEEE 802.15.7 [20] provides dimming and flicker control schemes for VLC.
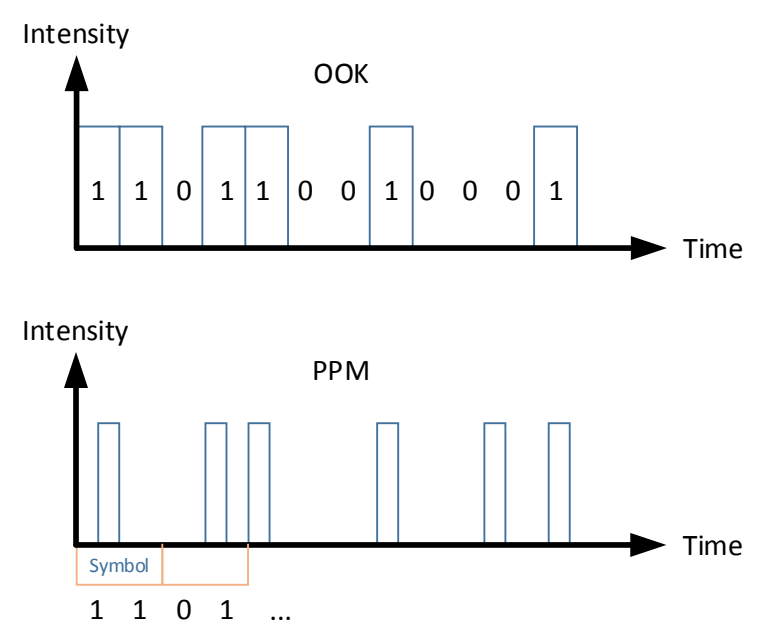

Figure 3: OOK and PPM techniques.

There are a number of modulation schemes that are proposed for VLC. We could not include all of them in this survey, but we review the most common and promising ones. As in Figure 3, On-off keying (OOK) is the simplest modulation technique for VLC, but this modulation scheme does not provide dimming control and does not support high data rates. Pulse position can be also used for modulation, which is called pulse-position modulation (PPM) as in Figure 3 [48]. PPM provides constant average power over time, thus flicker control can be achieved. To add dimming control, variable PPM (VPPM) is proposed as also mentioned in IEEE 802.15.7 [36]. In VPPM, pulse width is adjusted to control illumination level. However, PPM has spectral efficiency problems. To increase spectral efficiency of PPM, multi-pulse PPM (MPPM) is introduced. However, MPPM has problems with dimming control and data rate decreases significantly under low illumination [49]. To enhance MPPM, expurgated PPM (EPPM) [50] is proposed, in which, peak-power limit on sources are used. In EPPM, symbol length can be used for dimming control, but still the spectral efficiency is low as in PPM. To this end, multilevel EPPM (MEPPM) is proposed in [51], and it utilizes linear combination of EPPM to make mul- tiple amplitude levels as in pulse amplitude modulation (PAM). In this way, spectral efficiency and flicker control can be increased, but the link becomes more susceptible to shadowing and multi-path effects. In addition to PPM, pulse-width modulation (PWM) can be utilized as well. In [52], multiple LEDs are utilized to adjust the pulse width while achieving efficient dimming control. PWM can be also combined with OFDM which does not have dimming control. In this way, dimming control can be achieved in OFDM.

In addition to the pulse based modulation schemes, color based modulation schemes are promising for high data rate applications as also suggested in IEEE 802.15.7 [36]. In color based schemes, transmitters code information with color while satisfying static color requirements [53]. Information is coded to instantaneous intensity of LED in color intensity modulation (CIM) [54], but complexity of CIM is high. Color-shift keying (CSK) codes information to color combinations by keeping constant power level $[55,56]$. In this way, CSK can emit white light. Figure 4 presents $4-C S K$ modulation scheme. According to IEEE 802.15.7 [36], three colors can be utilized for CSK to provide 4-CSK, 8-CSK up to 16-CSK. In addition, [56] uses four color to further increase the constellation size up to 64 . However, CSK may cause color shift because the amplitude of current may be mis-adjusted at dimming control. This problem is solved in metameric modulation (MM) [57] by achieving constant color control. Since MM puts an additional constraint, size of the constellation cannot be increased as in CSK, but still MM can be used in high data rate systems.

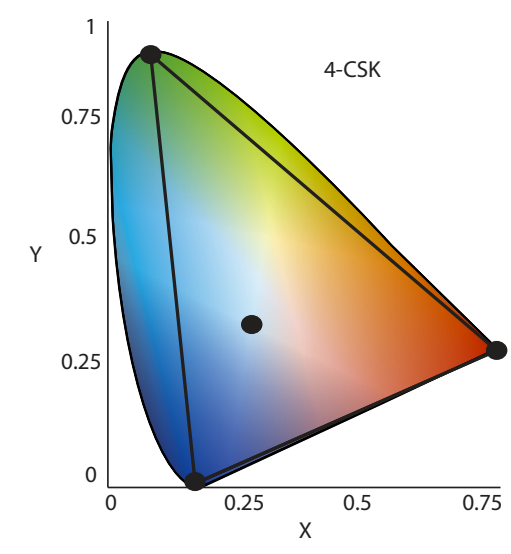

Figure 4: 4-CSK Modulation.

Spatial modulation (SM) systems can be also utilized in VLC [58]. In these systems, only one of the transmit arrays is active 
at a time as proposed recently in [59]. However, SM does not provide dimming control and has significant flicker effect [49].

Modulation bandwidth is one of most important challenges in VLC. Due to hardware imperfections and multi-path components, VLC suffers from the effects of inter-symbol interference (ISI) which can be solved with OFDM. To this end, we review OFDM techniques developed for VLC in the next subsection.

\section{3. $O F D M$}

VLC communication is generally provided via single LOS path. However, ISI is still a limiting factor in VLC due to the hardware imperfections [60]. As discussed in the previous subsection, conventional modulation schemes cannot support high speed applications due to limited bandwidth and high losses because intensity of light decreases significantly with distance. To alleviate effects of ISI, OFDM methods can be utilized for VLC [41, 61, 62]. In [63], OFDM with quadrature-amplitude modulation is utilized to reach $513 \mathrm{Mbps}$ with VLC. [64] provides a 1 Gbps broadband application of white LEDs with OFDM. [65] utilizes MIMO-OFDM employment to achieve data rates up to 1 Gbps. Dimming control can be provided by combining OFDM with PWM as introduced in [52]. Since VLC signals have to be both unipolar and real, conventional OFDM schemes, which use complex and bipolar signals, require some modifications.

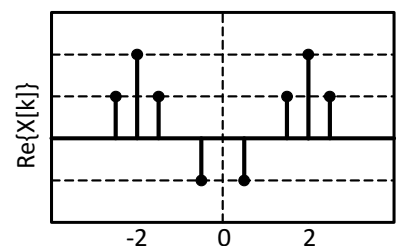

(a) Real part.

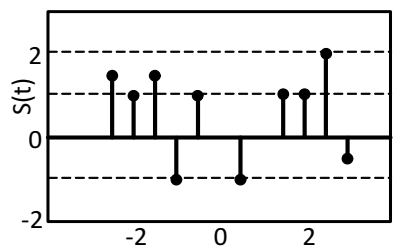

(c) Unbiased bipolar signal.

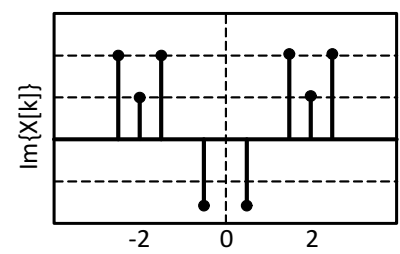

(b) Imaginary part.

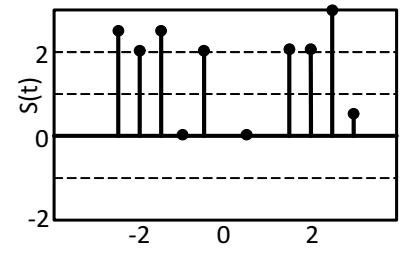

(d) Biased unipolar signal.
Figure 5: (a,b) OFDM signal with hermitian symmetry and, (c,d) addition of DC-bias for DCO-OFDM.

A number of OFDM schemes are proposed for VLC in order to provide unipolar and real time domain OFDM signals $[61,66,67,76,77,78,79]$. Most commonly, hermitian symmetry is utilized to generate real time signals at the expense of doubling the required bandwidth as in Figure 5(a) and Figure 5(b) [27]. In this way, the resulting real signal becomes bipolar, but it should be unipolar. For this purpose, direct current biased optical OFDM (DCO-OFDM) utilizes addition of direct current (DC)-bias to the bipolar signal to convert it to a unipolar signal as in Figure 5(c) and Figure 5(d) [66, 67]. The block diagram for DCO-OFDM is given in Figure 6. As in RF communications, the addition of DC-bias introduces high peak-to-average power ratio (PAPR). This high PAPR can be exploited for illumination purposes, but high DC-bias can adversely affect the communication performance because LEDs do not have a linear relationship between optical power and current. However, most applications utilize near linear region of LEDs [16]. Therefore, high DC-bias may make the system work on nonlinear region and create distortion by the amplifier. For these reasons, PAPR is a more significant problem in VLC compared to RF. In VLC, DFT-Spread OFDM is proposed as an effective way to eliminate this problem by utilizing localized DFT-Spread (LDFT-S) OFDM and interleaved DFT-Spread (IDFT-S) OFDM [60].

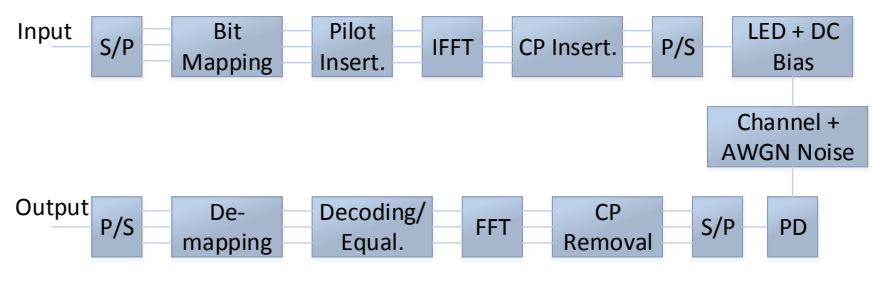

Figure 6: Block diagram of DCO-OFDM.

Another technique to avoid the DC bias is asymmetrically clipped optical OFDM (ACO-OFDM) which utilizes the properties of OFDM without requiring DC-biasing. In order to create a symmetric time domain signal, only the odd sub-carriers are used in ACO-OFDM [72]. In this way, negative values in signals are set to zero without altering carried information. Half of the spectrum is wasted in ACO-OFDM. For this reason, DCO-OFDM generally provides better spectral efficiency at the expense of power compared to ACO-OFDM [60].

To utilize all the spectrum, asymmetrically clipped DC biased optical OFDM (ADO-OFDM) is proposed in which ACOOFDM is utilized for odd subcarriers and DCO-OFDM is utilized for even subcarriers [73, 74]. In this way, entire spectrum is utilized in ADO-OFDM. ADO-OFDM requires less power compared to ADO-OFDM and ACO-OFDM. Detailed comparison of these techniques can be found in [73]. In [75], position modulating OFDM (PM-OFDM) is proposed by removing Hermitian symmetry constraint by utilizing DFT. Two receiver structures are designed in [75]. One achieves better BER performance compared to ACO-OFDM at the expense of high receiver complexity. There are a number of OFDM schemes which exploits different properties of the OFDM without biasing: pulse-amplitude-modulated discrete multitone modulation (PAM-DMT) [76], unipolar OFDM (U-OFDM) [77], flip-OFDM [78], spectrally-factorized optical OFDM (SFOOFDM) [79]. In addition, U-OFDM is extended to transmit multiple data frames in a single time domain signal in [80] as extended U-OFDM (eU-OFDM). eU-OFDM provides lower BER and PAPR compared to DCO-OFDM and U-OFDM.

To overcome PAPR problem, single carrier with frequency domain equalization (SCFDE) techniques are proposed: asymmetrically clipped optical (ACO-) SCFDE, repetition and clipping optical (RCO-) SCFDE, and decomposed quadrature optical (DQO-) SCFDE in [81]. SCFDE based modulation techniques have better BER and PAPR performance compared to 
previously discussed methods. [82] proposes on-off keying based SCFDE. In [83], pulse amplitude modulation (PAM)SCFDE is proposed to enable multiple order modulations. PAM-SCFDE outperforms PM-OFDM, DQO-SCFDE, ACOOFDM and eU-OFDM in terms of BER and PAPR in LOS and multipath links. Therefore, SCFDE based techniques are promising for visible light communications. Voltage clipping, dimming control and noise reduction are the major open research issues in SCFDE based modulation scheme.

To sum up, the comparison of the major modulation techniques proposed for VLC can be found in Table 3 [49]. According to our reviews, OFDM and color based modulation techniques are promising candidates for high data rate VLC applications. However, more research attention is required to solve the open research issues about transmitter and modulation techniques:

- Development of LEDs with more bandwidth and response time especially $\mu$ LEDs.

- Transmitter array design and optimization.

- Investigation and prevention of possible health related problems due to VLC.

- Designing and optimizing proposed modulation schemes for dimming and flicker control effectively.

\section{VLC Channel Modeling}

VLC channel modeling significantly depends on environment. For this reason, we review channel modeling for VLC for three main environments based on possible application areas: indoor, outdoor and underwater.

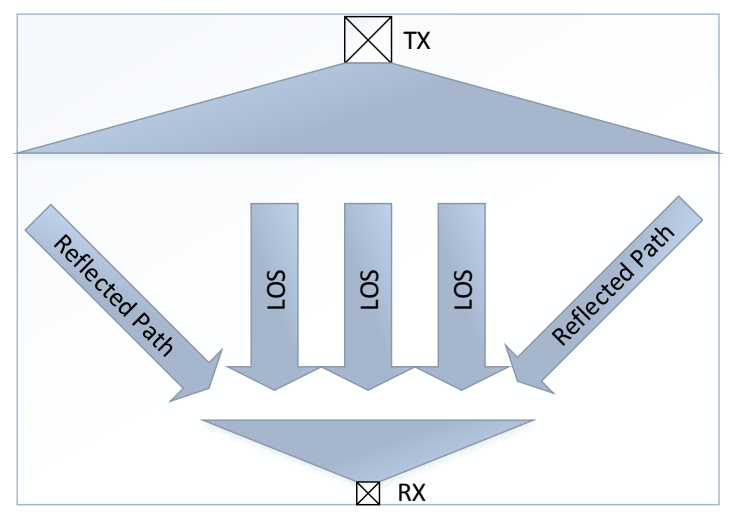

Figure 7: VLC light paths.

\subsection{Indoor}

VLC requires high SNR values for high data rate employments. For this reason, LOS paths are required for most of the applications. Therefore, indoor VLC channel modeling generally considers direct LOS and first order reflected diffuse paths as shown in Figure 7. In the early visible light communication studies $[10,12]$, visible light paths are modeled with the
Lambertian intensity based channel models developed for IR communications [84, 85]. However, IR communication channel models do not consider the dependence on wavelength in the reflections due to narrow-band nature of IR light sources. On the other hand, experimental studies have shown that visible spectrum reflections show dependence on the wavelengths [86]. To this end, [87, 88] propose a channel model which models the spectral reflectance in the visible spectrum to estimate more realistic channel characteristics for phosphor based white LEDs. Therefore, development of realistic channel models for other types of LEDs is an important open research issue because power spectral distribution of RGB LEDs and $\mu$ LEDs will be differ compared to white LEDs.

Another important issue in channel modeling is to model the noise in the system. The most important source of noise in VLC systems is the shot noise induced by ambient light. Shot noise $\left(\sigma_{\text {shot }}^{2}\right)$ in VLC represents white distribution [84]. In the absence of ambient noise, the fundamental source for the noise becomes the thermal noise at the receiver $\left(\sigma_{\text {thermal }}^{2}\right)$. At the end, noise plus interference in VLC can be given as

$$
N=\sigma_{\text {shot }}^{2}+\sigma_{\text {thermal }}^{2}+\gamma^{2} P_{r I S I}^{2},
$$

where $\gamma$ is the detector responsivity and $P_{\text {rISI }}$ is the power of the ISI. The expressions for the shot and thermal noise can be found in [10, 84]. Utilization of high data rate VLC system generally focuses on indoor applications because there will be significant shot noise in outdoor environments due to sun light.

\subsection{Outdoor}

Outdoor VLC applications cover utilization of street lamps to broadcast data and vehicle-to-vehicle communication applications. For broadcasting data with street lamps, the same theories are valid as in indoor VLC channel modeling, but the reflected signal component will have considerably lower effect on outdoor applications due to higher distances. For vehicle-tovehicle VLC applications, car lights (brakes and headlights) or traffic lights can be utilized to broadcast information and high speed cameras are utilized as receivers [89, 90, 91, 92]. However, there is no theoretical channel model for vehicular VLC applications considering speed of the vehicles and atmospheric conditions such as rain, snow and fog. In addition, outdoor applications will have significant shot noise due to sun during day time. Therefore, reliability of such links with time of the day and atmospheric conditions are important open research areas.

\subsection{Underwater}

Visible light is considerably faster than acoustic waves and visible spectrum offers significantly higher bandwidths. Thus, VLC is promising for underwater applications as well [93, 94]. However, the visible light spectrum is strongly affected by absorbing and scattering effects of water. For this reason, underwater VLC channel can be modeled with extinction factor which represents the dependency of light intensity to distance $[93,95,96]$. By using extinction factor, power of VLC paths can be modeled with Lambertian intensity based diffuse channel modeling $[93,95]$. In addition, the vector radiative transfer theory is utilized to model dispersion effect of the water 
which creates multiple scattering and ISI [94]. Underwater VLC channels show significant dependence to characteristics of water such as temperature, salt level, etc. unlike indoor and outdoor channels.

The open research areas in VLC channel modeling can be listed as

- Development of realistic channel models for all types of LEDs.

- Reliability analysis for vehicular VLC applications.

- Determining effects of atmospheric conditions on VLC for outdoor applications.

- Channel modeling and reliability analysis for underwater links considering water properties and depth.

\section{VLC Receiver}

Optical receivers for VLC can be divided into two categories: high data rate receivers and low data rate receivers. Up to now, we have discussed high data rate applications in which photodetectors are utilized to detect the light. However, low data rate applications do not require such sensitive devices. Instead, they can be realized with cameras that most of the smart phones have. Low data rate applications mainly cover broadcasting of a simple text such as a web link for a product on store shelf. An application can be designed to detect such information with a camera which are already in use. On the other hand, high data rate applications require more complex detectors.

Optical receivers consist of a photo-detector (PD) and an optical element. PD is the element which converts radiation into photo-current. After that, photo-current may be pre- or postamplified [97]. The optical element can be a lens or optical concentrator for non-imaging systems. The area of PD should be as large as possible in order to provide high gains. In [98], single link can provide up to 3 Gbps for closely spaced transmitter and receiver. However, achieving a few Gbps transmission rates requires MIMO systems in a regular room applications. To this end, we review non-imaging and imaging type of optical MIMO systems.

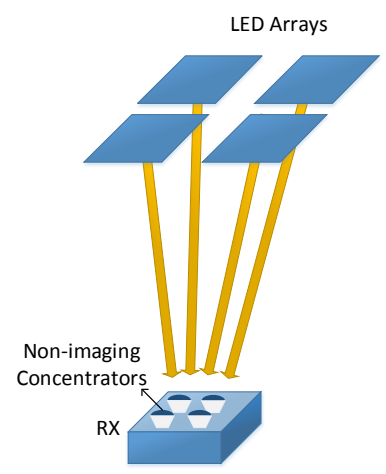

(a) Non-imaging

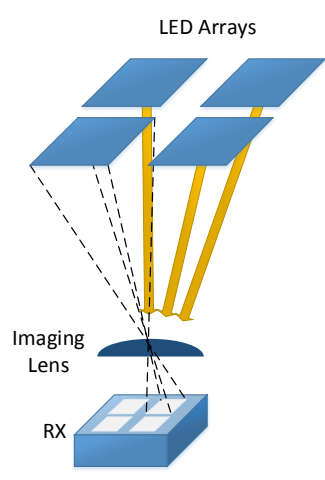

(b) Imaging
Figure 8: (a) Non-imaging and, (b) imaging optical MIMO systems.

\subsection{Non-imaging MIMO Systems}

Figure 8(a) shows the geometry of a non-imaging MIMO receiver. In this system, there is an optical concentrator above each receiver. In this way, a receiver can detect the light coming from every transmitter which is in range. For this reason, this employment resembles RF MIMO systems. However, there are two main disadvantages of non-imaging MIMO systems. The first one is the high correlation between receivers. Close spaced $\mathrm{RF}$ receivers (generally half a wavelength) have low correlation due to signal phase and rich scattering environment. However, light intensity does not show significant changes with time and close distance, thus VLC receivers become correlated. Second problem is rank of the channel gain matrix [32]. In MIMO systems, channel gain matrix should be full-rank, but if receiver unit is placed at the center of the transmitters or along the axes, the symmetry prevents the channel gain matrix to be full rank in non-imaging receivers. Additionally, unconstrained movement of users can make the channel gain matrix ill conditioned. In this way, BER can be significantly increased. On the other hand, imaging type receivers use a single optical concentrator to solve this problem as will be reviewed in the next subsection.

Although non-imaging type of receivers have some problems, they still provide higher receive coverage compared to imaging receivers [58]. For this purpose, [27] introduces a nonimaging angle-diversity receiver as shown in Figure 9. With this design, a receiver can provide wide coverage thanks to the narrow-beam non-imaging elements. Angle diversity exploits directivity of light to solve the rank problem of the channel gain matrix. In this way, non-imaging receivers can be used in high capacity systems with high coverage.

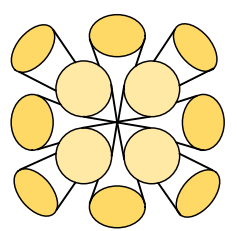

Figure 9: Angle-diversity receiver.

\subsection{Imaging MIMO Systems}

In imaging MIMO systems, receivers have a single optical concentrator as shown in Figure 8(b). The optical concentrator separates lights coming from different transmitter arrays. The images of different transmitter arrays may be intersecting according to path geometry. In addition, blurring and misfocus may create unwanted loss. However, optical concentrator provides decorrelated images. To this end, [99] proposes an imaging receiver with a hemispherical lens to provide $70^{\circ}$ coverage. [100] provides experimental results for imaging receivers in terms of coverage and BER. In imaging systems, receivers have more number of elements than transmitters in order to provide full rank channel gain matrix [32]. Therefore, imaging receivers can be used in high data rate applications [42, 65]. In [101], integrated CMOS based imaging MIMO receiver achieves 920 
Mbps by using OOK modulation scheme. By using more complex modulation schemes as discussed in Section 4.2, imaging receivers can achieve Gbps data rates.

Although imaging type of systems have certain benefits, location of user is very important because imaging lens may not cover all of the room. In this way, light coming from some transmitter arrays cannot be received. Therefore, there is a trade-off between imaging and non-imaging receivers in terms of coverage and data rates. To this end, open research issues regarding VLC receivers can be listed as

- Designing efficient MIMO receivers to solve the geometry dependencies.

- Combining non-imaging and imaging receivers to utilize both of their benefits.

\section{Medium Access Control Layer, Network Layer and Mul- tiple Access Techniques}

In this section, we first focus on medium access and network layers in VLC. Then, we examine the limited work on multiple access in VLC.

\subsection{Medium Access Control and Network Layers}

Research on MAC layer for VLC is rather limited. A fullduplex VLC implementation that uses carrier sense multiple access with collision detection (CSMA/CD) was proposed in [102]. For the uplink, IR was used. CSMA/CD was chosen for compatibility with Ethernet networks and PLC. The European Community Home Gigabit Access project (OMEGA) [103] aimed at providing a proof-of-concept ultra-broadband home area network. The MAC layer provided support for mobility, allowing multiple users to move from one access point to another.

Ambient light noise and shadowing are two very important factors that affect the performance of MAC operation considerably. In [104], authors investigate the feasibility of a vehicular VLC system. They point out that vehicular networks are exposed to both diurnal and nocturnal ambient noise and investigate the system performance of LED light sources under ambient noise. They also investigate the feasibility of fullduplex transmission in vehicular networks. The scattering from one direction is experienced as additional ambient noise for the receiver on the other side, and there is a certain performance degradation. They conclude that full duplex operation is not feasible for distances greater than $1.5 \mathrm{~m}$. To avoid the adverse effect of shadowing on users' data rate, a polling protocol that avoids assigning channels with shadowing to users was proposed in [105].

In [106], a LED-to-LED VLC system is proposed where LEDs are used as receiver instead of PDs. A CSMA with collision avoidance (CSMA(CA) approach is employed. Authors report that throughput decreases with increasing number of VLC devices. This is due to the increasing number of colliding frames. However, this method may still be considered since VLC systems are generally envisioned as broadcasting systems where the number of transmitters per receiver will be limited to only one or two.

With this type of operation, routing is generally not a complex issue. Communication inside the VLC domain is generally single hop - from the serving lighting device to the user placed under it. However, there are cases where routing must be considered for VLC. One such case is vehicular VLC, where communication between vehicles is done via head and tail lights of the vehicles. In such cases multi-hop VLC connections are possible. In [104], the performance of Ad hoc On-Demand Distance Vector Routing (AODV) protocol is given for a scenario case, where VLC is used in vehicle-to-roadside communication. Authors report that VLC can take advantage of its higher spatial use and perform better in dense traffic scenarios. The higher spatial reuse is due to two factors. First, the range of VLC is shorter. Second, vehicles block light, and this enables use of same frequencies by other vehicles. It is also noted that AODV has high delay in finding new routes and new routing schemes for VLC may yield better results. However, the authors do not present any such scheme.

In addition to such unicast routing schemes, multicast schemes are also necessary for vehicular communication. A multicast type more used in vehicular networks is geocast, which is mostly used to disseminate data within a certain area to inform drivers of recent road conditions and accidents for collision warning and avoidance. There are various geocast routing schemes in the literature. However these are developed for conventional wireless links. VLC has its own advantages (e.g. higher frequency resue and higher bandwidth), and disadvantages (e.g. shorter range, performance degradation in daylight). Therefore, novel routing schemes that take these into consideration are needed.

Another issue specific to VLC is flicker control. To this end, RLL line codes are used, as mentioned in Section 3. Clock and Data recovery (CDR) requires hard decisions in VLC. Therefore, it is important to choose appropriate forward error correction (FEC) codes that work well with hard decisions. In 802.15.7 Reed-Solomon (RS) and convolutional codes (CC) are used since these support hard decision decoding and interact well with RLL. Errors detected from the RLL line code at the receiver is marked as erasures to the RS decoder. This provides performance improvements of around $1 \mathrm{~dB}$ [22].

\subsection{Multiple Access Techniques}

Point-to-point communication up to hundreds of Mbps has been demonstrated in VLC. The next aim is to establish an efficient networking solution. Multiple access schemes are essential to allow multiple users share the communication medium. Certain modifications must be done to conventional multiple access schemes to be able to use them in VLC. Studies on multiple access schemes for VLC indicate CDMA is very inefficient since unipolar signals create considerable interchannel interference (ICI) [107]. The transmit power requirement is also much higher compared to the RF case. TDMA and OFDMA yield similar performances but OFDMA has higher power requirement. Since OFDMA has a wider time domain signal distribution, the DC biasing levels need to be higher. This is the reason 
for the higher power requirement. However, since this DC biasing will be used for illumination in VLC, the power difference is not a significant factor.

Aside from these conventional multiple access methods, there are some methods that are specific to VLC, or work well in VLC. Discrete multi-tone (DMT) modulation is an efficient single-transmitter technique for visible-light communication. In [108], authors investigate a discrete multi-tone multiple access (DMT-MA) system. It is closely related to OFDMA. It is possible to allocate and modulate each sub-carrier adaptively, according to channel conditions, under a total power constraint. A heuristic-based resource allocation algorithm is proposed which enables interference-aware allocation of sub-carriers to users. The proposed method improves VLC throughput especially when the number of sources in the room gets higher. However, it has problems with mobility, since the method requires traffic that does not change within a set of DMT symbols.

An optical code-division multiple access system (OCDMA) is presented in [109]. The method uses random optical codes. These codes do not have optimal correlation properties, but they are easier to generate. Authors argue that, as the number of users increase, the degradation caused by the non-optimality diminishes. However, VLC is generally characterized by small number of users per transmitter. A space-division multiple access (SDMA) scheme that uses optical beamforming to accommodate multiple users is presented in [110]. A setup that demonstrates a two user system is presented. Up to $12 \mathrm{~dB}$ improvement on the received signal for both channels were reported. However, this improvement is for a transmission distance shorter than $1 \mathrm{~m}$.

Handover is another important issue. The research so far is limited to power adjustment methods for handover [29], extensions to IEEE 802.15.7 for handover support [111], and cell zooming methods [112]. RF-hybrid systems that utilize WiFi when a VLC link is not available have also been considered [113]. In VLC, cell sizes are considerably smaller due to high directivity of light and smaller transmission distances. This causes received power to fluctuate more when the receiver is in motion. Soft handover mechanisms are especially important to even out these fluctuations and maintain a more stable connection. However, there is limited research on soft handover methods designed specifically for VLC. In [114], two soft handover methods are proposed and their performance is compared with hard handover. Presented results indicate that the proposed solutions provide higher data rate for both the overall system and individual users in the handover region.

The open research issues related to MAC, network layers and multiple access techniques can be listed as follows:

- Unicast and multicast routing algorithms for vehicular VLC is needed.

- Soft handover methods tailored to VLC.

- MAC protocols that take environmental lighting interference and shadowing into account

- Novel multiple access techniques for VLC. TDMA and CDMA perform poorly in non-flat channels. OFDMA has peak power problems, especially since DC bias is used in VLC because signals cannot be negative.

- Multiple access techniques for the uplink are needed.

\section{Potential Application Areas of VLC}

Initially, VLC was envisioned as an indoors communication technology. It can be used in homes to provide Internet access to residents. In Figure 10, we depict a possible scenario where VLC, Wi-Fi and PLC are used together to provide high speed connections to home users. Here, PLC is used as the backbone network that connects all light sources and enables their coordination.

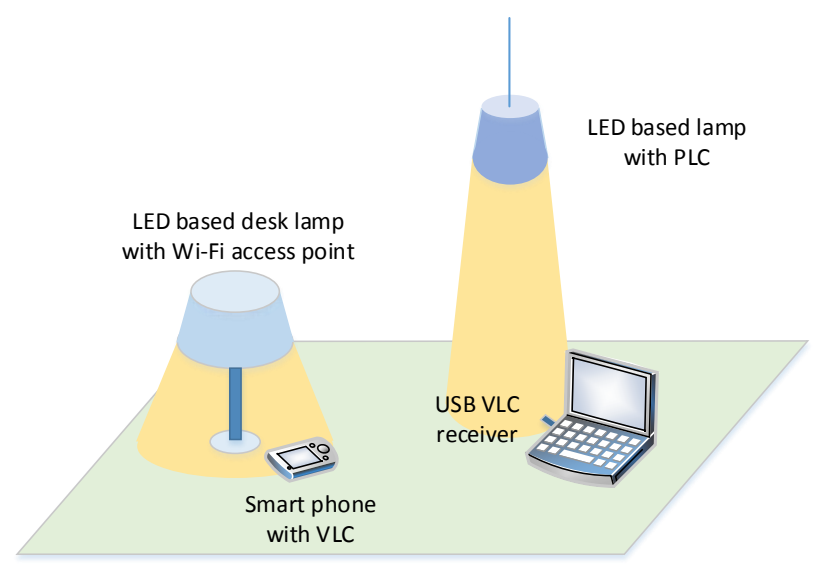

Figure 10: VLC powered intelligent homes.

Another application area is intelligent homes where VLC is readily available. One of the major concerns for machineto-machine (M2M) communications that enable smart home, smart city and on a larger scale Internet of Things (IoT) concepts is the huge bandwidth that will be used by the large number of sensors. VLC provides a very promising alternative to $\mathrm{RF}$ communication in this application area. However, for concepts like smart home to be realized via VLC, effective uplink communication methods are needed to enable sensors to send their data using VLC. In Figure 11(a), we depict a kitchen with intelligent sensors, communicating via VLC.

VLC can also be used in shopping malls to direct people to the products they are looking for. Product information such as pricing, safety remarks, consumer reviews, etc. can also be disseminated using VLC. Another indoors application area is information dissemination on exposition pieces in museums. Each light source illuminating specific pieces may transmit detailed information about the piece and visitors near that piece may use their VLC receivers to obtain this information.

VLC is also a very promising solution in application areas where information security is important. Since visible light is easily confined within a room, it is easy to provide required security with VLC. Military applications that require high security may benefit from this confinement property of VLC, rendering eavesdropping impossible. Furthermore, since visible light does not cause malfunctions in electronic devices, VLC 


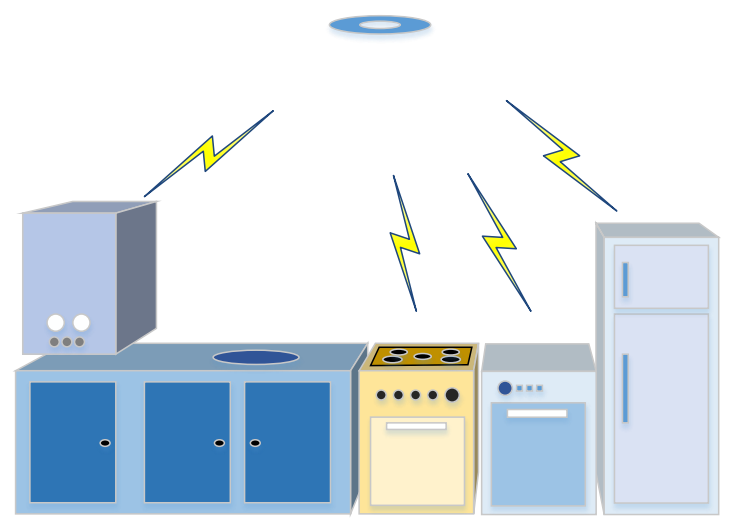

(a)

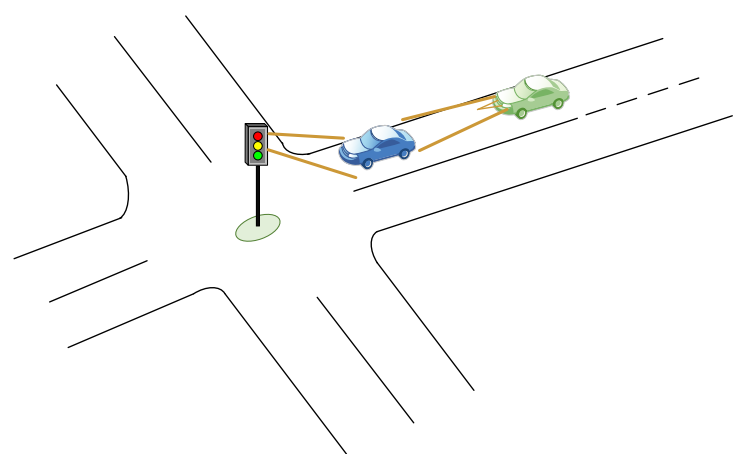

(b)

Figure 11: VLC applications (a) smart home M2M VLC, (b) vehicular VLC

can be employed in places where delicate electronic equipment is used. Hospitals can benefit from both of these advantages provided by VLC. Vital signs and other important information about patients must be monitored and it is important that the operation of these devices not be hampered by RF interference. Furthermore, patients' personal medical information must remain private. VLC provides both the required security and safety of devices. Another example where unhampered operation of delicate devices is required is chemical plants. Here, VLC can be used in conjunction with PLC to provide connectivity to the sensitive regions of the facility.

VLC can also be used in outdoor environments. One of the most promising application areas is vehicular communication. Car headlights are designed to be highly directive. Furthermore, cars move inside lanes, aligned with each other. Therefore, VLC can be used in car head and tail lights for vehicular communication. Also, street lights may be utilized for vehicular VLC as well. One of the financial concerns for infrastructurebased vehicular communication is the cost of the roadside units. However, since street lights are already deployed, VLC may provide a cheaper alternative to RF-based communication, removing the need for roadside RF units. VLC based delay tolerant routing schemes may be needed where vehicles may be used to receive and store information obtained from street lights of other vehicles via VLC and relay this information later when they are closer to the destination. Furthermore, traffic lights may also be used in vehicular VLC to report traffic conditions ahead, and relay important notifications [115, 116]. In Figure 11(b), we show a vehicular VLC scenario, where vehicles can communicate using head and tail lights, as well as traffic lights.

Underwater is another possible application area for VLC. Since RF waves attenuate very rapidly underwater, communication is conventionally performed via acoustic waves and the available bandwidth is low. VLC offers an alternative portion of the spectrum and larger bandwidth, resulting in faster data communication [93]. However, currently communication distance for underwater VLC is rather limited.

Since signboards and similar displays are generally assembled using LED arrays, VLC can be considered for advertising using visible light signboards [117]. In the entertainment industry, existing standards for lighting control, such as ANSI E1.11 - USITT DMX512-A [118] can be supplemented to enable VLC in theaters and other places of entertainment to disseminate information related to the ongoing show [119]. VLC can also be used to make interactive toys [120]. Toys can be controlled by a smartphone using VLC, where the phone's flash LED and camera are used as transmitter and receiver [121].

Finally, VLC can also be used as part of a positioning system [122]. Various approaches such as triangulation or proximity to a beacon are adopted [123, 124, 125].

- Methods that increase the limited communication range of VLC to open it up for further application areas.

- Schemes that increase mobility support of VLC.

- Uplink and/or full duplex solutions that enable VLC to be used in M2M more effectively by enabling sensors to report data using VLC.

- VLC based delay tolerant routing schemes for vehicular VLC.

\section{Conclusion}

In this paper, we provide a comprehensive survey of VLC as an alternative to RF communications. The available studies have shown that VLC can be used in high data rate applications in indoor communications. Therefore, VLC is a promising method to meet ever growing need for wireless access and data rate. Since VLC is a relatively new research area, there are many problems which require significant research attention. However, well-developed techniques for RF communications can be adapted to the characteristics of VL.

\section{Acknowledgment}

This work was supported by TURK TELEKOM. 


\section{References}

[1] Cisco Visual Networking Index, "Global Mobile Data Traffic Forecast Update, 2012-2017," White Paper, CISCO (Feb. 2013).

[2] Y-J Lin, H. A. Latchman, L. Minkyu, S. Katar, "A Power Line Communication Network Infrastructure for the Smart Home," IEEE Wireless Communications, vol.9, no.6, pp.104,111, Dec. 2002.

[3] A. Ahmed, J. A. Khan, U. Younis, "A High Speed Transmitter for Visible Light Communications upto $50 \mathrm{Mbps,"} \mathrm{International} \mathrm{Conference} \mathrm{on}$ Emerging Technologies (ICET), pp.153,157, 8-9 Dec. 2014.

[4] http://energy.gov/energysaver/articles/led-lighting

[5] T. Gilling, "The STREAM TONE: The Future of Personal Computing?," Troubador Publishing Ltd, 2015, ISBN 1784620815, 9781784620813.

[6] IEEE Std 1901-2010, "IEEE Standard for Broadband Over Power Line Networks: Medium Access Control and Physical Layer Specifications," 2010.

[7] ITU-T Rec. G.9960 and G.9961, "Unified High-Speed Wire-Line based Home Networking Transceivers," 2010.

[8] M. Hao, L. Lampe, S. Hranilovic, "Integration of Indoor Visible Light and Power Line Communication Systems," 17th IEEE International Symposium on Power Line Communications and Its Applications (ISPLC), 2013, pp.291,296, 24-27 March 2013

[9] "Lighting the Way: Perspectives on the Global Lighting Market," 2nd ed., McKinsey \& Company, 2012, http://www.mckinsey.com

[10] T. Komine, M. Nakagawa, "Fundamental Analysis for Visible-Light Communication System Using LED Lights," IEEE Transactions on Consumer Electronics, vol. 50, no. 1, Feb. 2004, pp.100-107, doi: 10.1109/TCE.2004.1277847.

[11] ISO 8995:2002(E)/CIE S 008/E-2001, "Lighting of Indoor Work Places"

[12] J. Grubor et al., "Bandwidth Efficient Indoor Optical Wireless Communications with White Light Emitting Diodes," Proc. 6th Intl. Symp. Commun. Sys., Networks and Digital Signal Proc., vol. 1, Graz, Austria, 23 25 June 2008, pp. 165-69.

[13] T. Komine, M. Nakagawa, "Integrated System of White LED VisibleLight Communication and Power-Line Communication," IEEE Transactions on Consumer Electronics, vol. 49, no. 1, pp.71-79, Feb. 2003 doi: 10.1109/TCE.2003.1205458.

[14] V. Chandrasekhar, J. Andrews, and A. Gatherer, "Femtocell Networks: A Survey," IEEE Commun. Mag., vol. 46, no. 9, 2008, pp. 59-6746, no. 9, pp. 59-67.

[15] W. Webb, "Wireless Communications: The Future", Wiley, 2007

[16] H. Burchardt, N. Serafimovski, D. Tsonev, S. Videv, H. Haas, "VLC: Beyond Point-to-Point Communication," IEEE Communications Magazine, vol. 52, no. 7, pp. 98-105, July 2014 doi: 10.1109/MCOM.2014.6852089.

[17] Li-Fi Consortium. http://lificonsortium.com/index.html

[18] Visible Light Communications Consortium, http://www.vlcc.net.

[19] M. Kavehrad, P. Amirshahi, "Hybrid MV-LV Power Lines and White Light Emitting Diodes for Triple-Play Broadband Access Communications," IEC Comprehensive Report on Achieving the Triple Play: Technologies and Business Models for Success, ISBN 1-931695-51-2, January 2006, pp. 167-178.

[20] IEEE Standard for Local and Metropolitan Area NetworksPart 15.7: Short-Range Wireless Optical Communication Using Visible Light, IEEE Std 802.15.7-2011

[21] B. Bai, Z. Xu, and Y. Fan, "Joint LED Dimming and High Capacity Visible Light Communication by Overlapping PPM," in: Proc. IEEE Annual Wireless and Opt. Commun. Conf., May 2010, pp. 71-75.

[22] S. Rajagopal, R. D. Roberts, S. Lim, "IEEE 802.15.7 Visible Light Communication: Modulation Schemes and Dimming Support," IEEE Communications Magazine, vol.50, no.3, March 2012, pp.72-82, doi: 10.1109/MCOM.2012.6163585.

[23] "500 Megabits/Second with White LED Light," (Press release) Siemens. Jan 18, 2010. Available at http://www.siemens.com/innovation/en/news/2010/500-megabitssecond-with-white-led-light.htm

[24] “Axrtek MOMO," Axrtek Inc. http://axrtek.com/momo/

[25] IEEE 802.15 WPAN 15.7 Amendment - Optical Camera Communications Study Group (SG 7a). http://www.ieee802.org/15/pub/SG7a.html

[26] J. Armstrong, Y. Sekercioglu, A. Neild, "Visible Light Positioning: A Roadmap For International Standardization," IEEE Communications Magazine, vol.51, no.12, pp.68,73, December 2013.
[27] D. Tsonev, S. Videv, H. Haas, "Light Fidelity (Li-Fi): Towards All-Optical Networking," in: Proc. SPIE 9007, Broadband Access Communication Technologies VIII, 900702 (December 18, 2013); doi:10.1117/12.2044649.

[28] Lumileds:Luxeon design guides.www.lumileds.com. 2006.

[29] Cheng Chen, D. Tsonev, H. Haas, "Joint Transmission in Indoor Visible Light Communication Downlink Cellular Networks," IEEE Globecom Workshops (GC Wkshps), 9-13 Dec. 2013, pp. 1127-1132.

[30] Occupational-Safety-and-Health-Branch-Labour-Department, "Simple Guide to Health Risk Assessment - Office Environment Series OE 2/99," 1998.

[31] Lubin Zeng, D. O’Brien, Hoa Le-Minh, Kyungwoo Lee, Daekwang Jung, Yunje Oh, "Improvement of Date Rate by Using Equalization in an Indoor Visible Light Communication System," IEEE International Conference on Circuits and Systems for Communications, pp. 678-682, 26-28 May 2008.

[32] Lubin Zeng, D. O'brien, Hoa Minh, G. Faulkner, Kyungwoo Lee, Daekwang Jung, Yunje Oh, Eun Tae Won, "High Data Rate Multiple Input Multiple Output (MIMO) Optical Wireless Communications Using White LED Lighting," IEEE Journal on Selected Areas in Communications, vol. 27, no. 9, December 2009, pp.1654-1662.

[33] K. Cui, J. Quan, and Z. Xu, "Performance of Indoor Optical Femtocell by Visible Light Communication,” Opt. Commun., vol. 298-299, July 2013, pp. 59-66.

[34] G. W. Marsh and J. M. Khan, "50-Mb/S Diffuse Infrared Free-Space Link Using On-Off Keying with Decision-Feedback Euqalisation," IEEE Photonics Technol. Lett., vol. 6, 1994, pp. 1268-1270.

[35] IEEE 802.15 WPAN Task Group 7 (TG7) Visible Light Communication. http://www.ieee802.org/15/pub/TG7.html

[36] IEEE Standard for Local and Metropolitan Area Networks — Part 16: Air Interface for Fixed Broadband Wireless Access Systems - Amendment 2: Medium Access Control Modifications and Additional Physical Layer Specifications for 2-11 GHz," IEEE Std 802.16a-2003 (Amendment to IEEE Std 802.16-2001), pp. 1-292, 2003

[37] J. J. D. McKendry, R. P. Green, A. E. Kelly, Zheng Gong, B. Guilhabert, D. Massoubre, E. Gu, M. D. Dawson, "High-Speed Visible Light Communications Using Individual Pixels in a Micro Light-Emitting Diode Array," IEEE Photonics Technology Letters, vol. 22, no. 18, Sep. 2010, pp. 1346-1348.

[38] S. Zhang, S. Watson, J. J. D. McKendry, D. Massoubre, A. Cogman, E. Gu, R. K. Henderson, A. E. Kelly, M. D. Dawson "1.5 Gbit/s MultiChannel Visible Light Communications Using CMOS-Controlled GaNBased LEDs," Journal of Lightwave Technology, vol. 31, no. 8, pp. 12111216, April15, 2013.

[39] J. W. Shi, J. K. Sheu, C. H. Chen, G. R. Lin, and W. C. Lai, "High-Speed GaN-based Green Light-Emitting Diodes with Partially N-Doped Active Layers And Current-Confined Apertures,' IEEE Electron Device Lett., vol. 29, no. 2, Feb. 2009, pp. 158-160.

[40] J. Vucic, C. Kottke, S. Nerreter, A. Buttner, K.-D. Langer, and J. W. Walewski, "White Light Wireless Transmission at 200+ Mb/s Net Data Rate by Use of Discrete-Multitone Modulation," IEEE Photon. Technol. Lett., vol. 21, no. 20, Oct. 15, 2009, pp. 1511-1513.

[41] A. Shaw, A. Bradley, J. Donegan, and J. Lunney, "GaN Resonant Cavity Light-Emitting Diodes For Plastic Optical Fiber Applications," IEEE Photon. Technol. Lett., vol. 16, no. 9, Sep. 2004, pp. 2006-2008.

[42] Hao Le Minh, D. O’Brien, G. Faulkner, Lubin Zeng, Kyungwoo Lee, Daekwang Jung, Yunje Oh, "High-Speed Visible Light Communications Using Multiple-Resonant Equalization,' IEEE Photonics Technology Letters, vol. 20, no. 14, July15, 2008, pp. 1243-1245.

[43] A. Burton, Z. Ghassemlooy, S. Rajbhandari, and S.-K. Liaw, ”Design and Analysis of an Angular-Segmented Full-Mobility Visible Light Communications Receiver," Transactions on Emerging Telecommunications Technology, Mar. 2014.

[44] A. Nuwanpriya, S.-W. Ho and C. S. Chen, "Angle Diversity Receiver for Indoor MIMO Visible Light Communications", in Proc. IEEE Globecom Workshops (Optical Wireless Communications), Austin, USA, pp. 529534, 2014.

[45] A. Nuwanpriya, S.-W. Ho and C. S. Chen, "Indoor MIMO Visible Light Communications: Novel Angle Diversity Receivers for Mobile Users", IEEE Journal on Selected Areas in Communications, vol. 33, pp. 1-13, August, 2015 
[46] A. Wilkins, J. Veitch, B. Lehman, "LED Lighting Flicker and Potential Health Concerns: IEEE standard PAR1789 Update," Energy Conversion Congress and Exposition (ECCE), 2010 IEEE, pp. 171-178, 12-16 Sept. 2010

[47] X. Bao, G. Yu, J. Dai, X. Zhu, "Li-Fi: Light Fidelity-a Survey,” Wireless Networks, 2015 doi:10.1007/s11276-015-0889-0.

[48] M. D. Audeh, J. M. Kahn, "Performance Evaluation of L-Pulse-Position Modulation on Non-Directed Indoor Infrared Channels," Communications, 1994. ICC '94, SUPERCOMM/ICC '94, Conference Record, 'Serving Humanity Through Communications.' IEEE International Conference on, pp. 660-664 vol.2, 1-5 May 1994.

[49] M. Noshad and M. Brandt-Pearce, "Can Visible Light Communications Provide Gb/s Service?,” arXiv:1308.3217, pp. 17, Aug. 2013.

[50] M. Noshad and M. Brandt-Pearce, "Expurgated PPM Using Balanced Incomplete Block Designs," IEEE Commun. Lett., vol. 16, no. 7, pp. 968971, 2012

[51] M. Noshad, M. Brandt-Pearce, "Multilevel Pulse-Position Modulation Based on Balanced Incomplete Block Designs," Global Communications Conference (GLOBECOM), 2012 IEEE, pp. 2930-2935, 3-7 Dec. 2012.

[52] G. Ntogari, T. Kamalakis, J. Walewski, and T. Sphicopoulos, "Combining Illumination Dimming Based on Pulse-Width Modulation with VisibleLight Communications Based on Discrete Multitone,IEEE J. Opt. Commun. Netw., vol. 3, no. 1, pp. 5665, 2011.

[53] CIE (1931). Commission Internationale de lEclairage proceedings, Cambridge University Press.

[54] K.-I. Ahn, J.K. Kwon, "Color Intensity Modulation for Multicolored Visible Light Communications," IEEE Photonics Technology Letters, vol. 24, no. 24, pp. 2254-2257, Dec.15, 2012.

[55] E. Monteiro, S. Hranilovic, "Design and Implementation of Color-Shift Keying for Visible Light Communications," Journal of Lightwave Technology, vol. 32, no. 10, pp. 2053-2060, May15, 2014

[56] R. Singh, T. O’Farrell, J. P. R. David, “An Enhanced Color Shift Keying Modulation Scheme for High-Speed Wireless Visible Light Communications," Journal of Lightwave Technology, vol. 32, no. 14, pp. 2582-2592, July15, 152014.

[57] P. M. Butala, J. C. Chau, T. D. C. Little, "Metameric Modulation for Diffuse Visible Light Communications with Constant Ambient Lighting,' Optical Wireless Communications (IWOW), 2012 International Workshop on, pp. 1-3, 22-22 Oct. 2012

[58] H. Elgala, R. Mesleh, H. Haas, "Indoor Optical Wireless Communication: Potential And State-of-the-Art," IEEE Communications Magazine, vol. 49, no. 9, Sep. 2011, pp. 56-62.

[59] R. Mesleh et al. , "Indoor MIMO Optical Wireless Communication Using Spatial Modulation," IEEE ICC 10, Cape Town, South Africa, 22-27 May 2010, pp. 1-5.

[60] Chaopei Wu, Hua Zhang, Wei Xu, "On Visible Light Communication Using LED Array with DFT-Spread OFDM,’ IEEE International Conference on Communications (ICC), 10-14 June 2014, pp. 3325-3330.

[61] Y. Tanaka, T. Komine, S. Haruyama, M. Nakagawa, "Indoor Visible Communication Utilizing Plural White LEDs as Lighting," in: IEEE International Symposium on Personal, Indoor and Mobile Radio Communications (PIMRC), San Diego, CA, Sept. 2001, pp. F81-F85.

[62] M. Z. Afgani, H. Haas, H. Elgala, D. Knipp, "Visible Light Communication Using OFDM," International Conference on Testbeds and Research Infrastructures for the Development of Networks and Communities (TRIDENTCOM), 2006

[63] J. Vucic, C. Kottke, S. Nerreter, K.-D. Langer, J. W. Walewski, "513 Mbit/s Visible Light Communications Link Based on DMT-Modulation of a White LED," Journal of Lightwave Technology, , vol. 28, no. 24, Dec.15, 2010, pp. 3512-3518.

[64] A. M. Khalid, G. Cossu, R. Corsini, P. Choudhury and E. Ciaramella, "1-Gb/s Transmission Over a Phosphorescent White LED by Using RateAdaptive Discrete Multitone Modulation,” IEEE Photonics Journal, Oct. 2012, pp. 1465-1473.

[65] A. H. Azhar, T. Tran, D. O’Brien, "A Gigabit/s Indoor Wireless Transmission Using MIMO-OFDM Visible-Light Communications," IEEE Photonics Technology Letters, vol. 25, no. 2, Jan. 15, 2013, pp. 171-174.

[66] J. Armstrong, B. J. Schmidt, "Comparsion of Asymmetrically Clipped Optical OFDM and DC-biased Optical OFDM in AWGN," IEEE Communications Letters, vol. 12, 2008, pp. 343-345.

[67] S. K. Hashemi, Z. Ghassemlooy, L. Chao, D. Benhaddou, "Orthogonal
Frequency Division Multiplexing for Indoor Optical Wireless Communications Using Visible Light LEDs," International Symposium on Communication Systems, Networks and Digital Signal Processing (CNSDSP), 25-25 July 2008, pp. 174-178.

[68] S. C. J. Lee, S. Randel, F. Breyer, A. M. J. Koonen, "PAM-DMT for Intensity-Modulated and Direct Detection Optical Communication Systems," IEEE Photonics Technology Letters 21, Dec. 2009 pp. 1749-1751.

[69] D. Tsonev, S. Sinanovic, H. Haas, "Novel Unipolar Orthogonal Frequency Division Multiplexing (U-OFDM) for Optical Wireless," in: Proc. of the IEEE Vehicular Technology Conference (VTC Spring), Yokohama, Japan, May 6-9 2012.

[70] N. Fernando, Y. Hong, E. Viterbo, "Flip-OFDM for Optical Wireless Communications," in: IEEE Information Theory Workshop (ITW), Paraty, Brazil, Oct., 16-20 2011.

[71] K. Asadzadeh, A. Farid, S. Hranilovic, "Spectrally Factorized Optical OFDM," in: 12th IEEE Canadian Workshop on Information Theory (CWIT 2011), 102-105, May 17-20 2011.

[72] J. Armstrong, A. Lowery, "Power Efficient Optical OFDM," IEEE Electronics Letters, vol. 42, no. 6, Mar. 16, 2006, pp. 370-72.

[73] S. D. Dissanayake, J. Armstrong, "Comparison Of ACO-OFDM, DCOOFDM And ADO-OFDM in IM/DD Systems", Journal Of Lightwave Technology, vol. 31, no. 7, April 1, 2013.

[74] S. D. Dissanayake, K. Panta, and J. Armstrong, "A Novel Technique to Simultaneously Transmit ACO-OFDM and DCO-OFDM in IM/DD Systems," in Proc. IEEE GLOBECOM Workshops, Houston, TX, USA, 2011, pp. 782786

[75] A. Nuwanpriya, A. Grant, S.-W. Ho, and L. Luo, "Position Modulating OFDM for Optical Wireless Communications," in Proc. IEEE Globecom Workshops (Optical Wireless Communications), 2012, pp. 12191223.

[76] S. C. J. Lee, S. Randel, F. Breyer, A. M. J. Koonen, "PAM-DMT for Intensity-Modulated and Direct Detection Optical Communication Systems," IEEE Photonics Technology Letters 21, Dec. 2009 pp. 1749-1751.

[77] Tsonev, D., Sinanovic, S., and Haas, H., "Novel Unipolar Orthogonal Frequency Division Multiplexing (U-OFDM) for Optical Wireless," in: Proc. of the IEEE Vehicular Technology Conference (VTC Spring), Yokohama, Japan, May 6-9 2012.

[78] N. Fernando, Y. Hong, E. Viterbo, "Flip-OFDM for Optical Wireless Communications," in: IEEE Information Theory Workshop (ITW), Paraty, Brazil, Oct., 16-20 2011.

[79] K. Asadzadeh, A. Farid, S. Hranilovic, "Spectrally Factorized Optical OFDM," in: 12th IEEE Canadian Workshop on Information Theory (CWIT 2011), 102-105, May 17-20 2011.

[80] D. Tsonev and H. Haas, "Avoiding Spectral Efficiency Loss in Unipolar OFDM for Optical Wireless Communication” in Proc. IEEE ICC, 2014, pp. 33423347

[81] K. Acolatse, Y. Bar-Ness, and S. K. Wilson, "Novel Techniques of Single Carrier Frequency-Domain Equalization for Optical Wireless Communications," EURASIP Journal on Advances in Signal Processing, vol. 2011, pp. 113, 2011.

[82] A. Nuwanpriya, J. A. Zhang, A. Grant, S.-W. Ho, and L. Luo, "Single Carrier Frequency Domain Equalization Based on On-Off-Keying For Optical Wireless Communications", in Proc. IEEE Wireless Communications and Networking Conference, Shanghai, China, pp. 3922-3927, 2013.

[83] A. Nuwanpriya, S. Ho, J. A. Zhang, A. Grant, L. Luo, "PAM-SCFDE for Optical Wireless Communications," Journal of Lightwave Technology, vol. 33, no. 14, pp. 2938-2949, July15, 2015.

[84] J. M. Kahn, J. R. Barry, "Wireless Infrared Communications," Proceedings of the IEEE, vol. 85, no. 2, Feb 1997, pp. 265-298.

[85] F. R. Gfeller, U. H. Bapst, "Wireless in-House Data Communication via Diffuse Infrared Radiation,” Proc. IEEE, vol. 67, Nov. 1979, pp. 14741486.

[86] K. Cui, G. Chen, Q. He, and Z. Xu, "Indoor Optical Wireless Communication by Ultraviolet snd Visible Light," SPIE, vol. 7464, Aug. 2009.

[87] K. Lee, H. Park, J. R. Barry, "Indoor Channel Characteristics for Visible Light Communications, IEEE Communications Letters, vol. 15, no. 2, pp. 217-219, February 2011.

[88] K. Lee, H. Park, "Channel Model and Modulation Schemes for Visible Light Communications, 2011 IEEE 54th International Midwest Symposium on Circuits and Systems (MWSCAS), pp. 1-4, 7-10 Aug. 2011.

[89] S. Iwasaki, C. Premachandra, T. Endo, T. Fujii, M. Tanimoto, Y. Kimura, 
"Visible Light Road-to-Vehicle Communication Using High-Speed Camera," IEEE Intelligent Vehicles Symposium, pp. 13-18, 4-6 June 2008

[90] S. Okada, T. Yendo, T. Yamazato,T. Fujii, M. Tanimoto, Y. Kimura, "OnVehicle Receiver for Distant Visible Light Road-to-Vehicle Communication,” IEEE Intelligent Vehicles Symposium, pp. 1033-1038, 3-5 June 2009.

[91] S. Arai, S. Mase, T. Yamazato, T. Endo, T. Fujii, M. Tanimoto, K. Kidono, Y. Kimura, Y. Ninomiya, "Experimental on Hierarchical Transmission Scheme for Visible Light Communication using LED Traffic Light and High-Speed Camera," IEEE Vehicular Technology Conference, pp. 21742178, Sept. 30 2007-Oct. 32007.

92] T. Nagura, T. Yamazato, M. Katayama, T. Yendo, T. Fujii, H. Okada, "Improved Decoding Methods of Visible Light Communication System for ITS Using LED Array and High-Speed Camera," IEEE Vehicular Technology Conference (VTC 2010-Spring), pp. 1-5, 16-19 May 2010.

[93] H. Uema, T. Matsumura, S. Saito, Y. Murata, "Research and Development on Underwater Visible Light Communication Systems," Electronics and Communications in Japan, vol. 98, no. 3, 2015, pp. 9-13.

94] S. Jaruwatanadilok, "Underwater Wireless Optical Communication Channel Modeling and Performance Evaluation using Vector Radiative Transfer Theory," IEEE Journal on Selected Areas in Communications, vol.26, no.9, pp. 1620-1627, December 2008.

[95] J. W. Giles and I. N. Bankman, "Underwater Optical Communications Systems. Part 2: Basic Design Considerations," IEEE Military Communications Conference, Atlantic City, NJ, USA, pp. 17005, 2005

[96] N. Fair, A. D. Chave, L. Freitag, J. Preisig, S. N. White, D. Yoerger, and F. Sonnichsen, "Optical Modem Technology for Seafloor Observatories,' OCEAN, Boston, MA, USA, 2006.

[97] D. O’brien, L. Zeng, Hoa Le-Minh, G. Faulkner, J. W. Walewski, S. Randel, "Visible Light Communications: Challenges and Possibilities," in: Proc. IEEE Personal, Indoor and Mobile Radio Communications Conference, 15-18 Sept. 2008, pp.1-5.

[98] Markets and Markets, "Visible Light Communication (VLC)/Li-Fi Technology \& Free Space Optics (FSO) Market (2013-2018)," tech. rep., Jan. 2013.

[99] T. Wang, Y. Sekercioglu, and J. Armstrong, "Analysis of an Optical Wireless Receiver Using a Hemispherical Lens with Application in MIMO Visible Light Communications," Journal of Lightwave Technology, vol. 31, pp. 1744-1754, Jun. 2013

[100] K. D. Dambul, D. O'Brien, and G. Faulkner, "Indoor Optical Wireless MIMO System with an Imaging Receiver," IEEE Photonics Technology Letters, vol. 23, pp. 97-99, Nov. 2011.

[101] S. Rajbhandari, H. Chun, G. Faulkner, K. Cameron, A. V. N. Jalajakumari, R. Henderson, D. Tsonev, M. Ijaz, Z. Chen, H. Haas, E. Xie, J. J. D. McKendry, J. Herrnsdorf, E. Gu, M. D. Dawson, D. O’Brien, "ImagingMIMO Visible Light Communication System Using LEDs and Integrated Receiver," in Proc. of Globecom Workshops 2014, pp. 536-540, 8-12 Dec. 2014

[102] X. Lin, K. Ikawa, K. Hirohashi, "High-speed Full-Duplex Multiaccess System for LED-Based Wireless Communications Using Visible Light,' the 2nd International Multi-Conference on Engineering and Technological Innovation, 2009.

[103] Home Gigabit Access (OMEGA) project http://www.ictomega.eu/home.html

[104] C. B. Liu, B. Sadeghi, E. W. Knightly, "Enabling Vehicular Visible Light Communication (V2LC) Networks," in Proc. VANET'11, Eighth ACM International Workshop on Vehicular inter-networking, pp. 41-50.

[105] J. Hou, D.C. O'Brien, D.J. Edwards, "Polling Scheme for Indoor LOS Optical Wireless LAN," Electronics Letters, 2003.

[106] S. Schmid, G. Corbellini, S. Mangold, T. R. Gross, "LED-to-LED Visible Light Communication Networks," in Proc. MobiHoc'13, Fourteenth ACM International Symposium on Mobile Ad hoc Networking and Computing, pp. 1-10

[107] D. Tsonev, S. Sinanovic, H. Haas, "Practical MIMO Capacity for Indoor Optical Wireless Communication with White LEDs," in: Proc. of the IEEE Vehicular Technology Conference (VTC Spring), 2-5 June 2013, pp. $1-5$.

[108] D. Bykhovsky, S. Arnon, "Multiple Access Resource Allocation in Visible Light Communication Systems," Journal of Lightwave Technology, vol. 32, no. 8, pp. 1594-1600, March 15, 2014.

[109] M.F. Guerra-Medina, B. Rojas-Guillama, O. Gonzalez, J.A. Martin-
Gonzalez, E. Poves, F.J. Lopez-Hernandez, "Experimental Optical CodeDivision Multiple Access System for Visible Light Communications," Wireless Telecommunications Symposium (WTS), 2011, pp.1,6, 13-15 April 2011

[110] S. Kim, H. Lee, "Visible Light Communication Based on SpaceDivision Multiple Access Optical Beamforming," Chin. Opt. Lett. 12, 120601-120601 (2014).

[111] T. Nguyen, Y. M. Jang, M. Z. Chowdhury, "A Pre-Scanning-based Link Switching Scheme in Visible Light Communication Networks," Ubiquitous and Future Networks (ICUFN), 2013 Fifth International Conference on, vol., no., pp.366,369, 2-5 July 2013

[112] M. B. Rahaim, T.D.C. Little, "SINR Analysis and Cell Zooming with Constant Illumination For Indoor VLC networks," Optical Wireless Communications (IWOW), 2013 2nd International Workshop on , vol., no., pp.20,24, 21-21 Oct. 2013

[113] M.B. Rahaim, A. M. Vegni, T.D.C. Little, "A Hybrid Radio Frequency and Broadcast Visible Light Communication system," GLOBECOM Workshops (GC Wkshps), 2011 IEEE , vol., no., pp.792,796, 5-9 Dec. 2011

[114] E. Dinc, O. Ergul, O. B. Akan, "Soft Handover in OFDMA based Visible Light Communication Networks," to appear in Proc. IEEE VTC 2015Fall, Boston, MA, USA, September 2015.

[115] N. Kumar et al., "Visible Light Communication for Intelligent Transportation in Road Safety Applications," Proc. Intl. Wireless Commun. and Mobile Computing Conf., 2011, pp. 1513-18.

[116] S. Kitano, S. Haruyama, and M. Nakagawa, "LED Road Illumination Communications System," in Proc. VTC-Fall, 2003, pp. 3346-3350.

[117] S.-B. Park, D. K. Jung, H. S. Shin, D. J. Shin, Y.-J. Hyun, K. Lee, Y. J. Oh, "Information Broadcasting System Based On Visible Light Signboard," in Proc. Wireless Opt. Commun. 2007, pp. 311-313.

[118] ANSI E1.11 2008 (R2013), Entertainment Technology - USITT DMX512-A, Asynchronous Serial Digital Data Transmission Standard for Controlling Lighting Equipment and Accessories.

[119] S-K Lim, K. Ruling, K. Insu, I. S. Jang, "Entertainment Lighting Control Network Standardization to Support VLC Services," IEEE Communications Magazine, vol.51, no.12, pp.42-48, December 2013.

[120] S. Schmid, G. Corbellini, S. Mangold, T. R. Gross, "Continuous Synchronization for LED-to-LED Visible Light Communication Networks," 3rd International Workshop in Optical Wireless Communications (IWOW), 2014, pp.45-49, 17-17 Sept. 2014.

[121] G. Corbellini, K. Aksit, S. Schmid, S. Mangold, T. Gross, "Connecting Networks of Toys And Smartphones With Visible Light Communication,' IEEE Communications Magazine, vol.52, no.7, pp.72,78, July 2014.

[122] Hyun-Seung Kim, Deok-Rae Kim, Se-Hoon Yang, Yong-Hwan Son, Sang-Kook Han, "An Indoor Visible Light Communication Positioning System Using a RF Carrier Allocation Technique," Journal of Lightwave Technology, vol. 31, no. 1, Jan.1, 2013, pp. 134-144.

[123] Hugh-Sing-Liu, Pang-G, "Positioning Beacon System Using Digital Camera and LEDs," IEEE Transactions on Vehicular Technology, 2003, 52, (2), pp.406-419

[124] X. Liu, H. Makino, S. Kobayashi, Y. Maeda, "Design of an Indoor SelfPositioning System for the Visually Impaired-Simulation with RFID and Bluetooth in a Visible Light Communication System," Engineering in Medicine and Biology Society, 2007. EMBS 2007. 29th Annual International Conference of the IEEE, 2007, pp. 1655-1658

[125] M. Yoshino, S. Haruyama, M. Nakagawa, "High-accuracy Positioning System Using Visible LED Lights And Image Sensor," IEEE Radio and Wireless Symposium, 2008, pp. 439-442. 